\title{
Predicate Introduction under Stable and Well-founded Semantics *
}

\author{
Johan Wittocx, Joost Vennekens, Maarten Mariën, Marc Denecker, and \\ Maurice Bruynooghe \\ Department of Computer Science, K.U. Leuven, Belgium \\ \{johan, joost, maartenm, marcd, maurice\}@cs.kuleuven. be
}

\begin{abstract}
This paper studies the tranformation of "predicate introduction": replacing a complex formula in an existing logic program by a newly defined predicate. From a knowledge representation perspective, such transformations can be used to eliminate redundancy or to simplify a theory. From a more practical point of view, they can also be used to transform a theory into a normal form imposed by certain inference programs or theorems, e.g., by eliminating universal quantifiers. In this paper, we study when predicate introduction is equivalence preserving under the stable and well-founded semantics. We do this in the algebraic framework of "approximation theory"; this is a fixpoint theory for non-monotone operators that generalizes all main semantics of various non-monotone logics, including Logic Programming, Default Logic and Autoepistemic Logic. We prove an abstract, algebraic equivalence result and then instantiate this abstract theorem to Logic Programming under the stable and well-founded semantics.
\end{abstract}

\section{Introduction}

This paper studies the tranformation of "predicate introduction" for Logic Programming. By this, we mean the introduction of a new predicate to be able to simplify the expressions in the bodies of certain rules. To motivate our interest in this transformation, we consider a slightly simplified version of part of a program that occurs in [1]. In this paper, a logic program (under the stable semantics) is constructed to capture the meaning of theories in the action language $\mathcal{A L}$. In particular, static causal laws of the following form are considered: " $P$ is caused if $P_{1}, \ldots, P_{N}$ ". Here, $P, P_{1}, \ldots, P_{N}$ are propositional symbols. (Throughout this paper, we will use the notational convention that predicates, functions, and constant symbols start with an upper case letter, while variables are all lower case.) In its logic programming translation, such a causal law $R$ is represented by the following set of facts: $\left\{\operatorname{Head}(R, P), \operatorname{Prec}\left(R, 1, P_{1}\right), \ldots\right.$, $\left.\operatorname{Prec}\left(R, N, P_{N}\right), N b O f \operatorname{Prec}(R, N)\right\}$.

Now, the meaning in $\mathcal{A L}$ of such a law is that whenever all of $P_{1}, \ldots, P_{N}$ hold, then so must $P$. Using the predicate Holds/1 to describe which propositions

\footnotetext{
* Works supported by FWO-Vlaanderen, IWT-Vlaanderen, and by GOA/2003/08.
} 
hold, this can be captured by the following rule (we use $\Leftarrow$ to represent material implication and $\leftarrow$ for the "rule construct" of Logic Programming):

$$
\forall p \operatorname{Holds}(p) \leftarrow \exists r \operatorname{Head}(r, p) \wedge \forall i \forall q \operatorname{Prec}(r, i, q) \Rightarrow \operatorname{Holds}(q) .
$$

This rule contains universal quantifiers $\forall$ in its body. Even though it is possible to define both stable and well-founded semantics for such programs, current model generation systems such as SModels or DLV cannot handle this kind of rules. As such, we would like to eliminate this quantifier. The well-known LloydTopor transformation [11] suggests introducing a new predicate, BodyNotSat/1, to represent the negation of the subformula $\phi=\forall i \forall q \operatorname{Prec}(r, i, q) \Rightarrow \operatorname{Holds}(q)$. Because $\neg \phi=\exists i \exists q \operatorname{Prec}(r, i, q) \wedge \neg \operatorname{Holds}(q)$, we would then get:

$$
\begin{aligned}
\forall p, r \operatorname{Holds}(p) & \leftarrow \operatorname{Head}(r, p) \wedge \neg \operatorname{BodyNotSat}(r) . \\
\forall r, i, q \operatorname{BodyNotSat}(r) & \leftarrow \operatorname{Prec}(r, i, q) \wedge \neg \operatorname{Holds}(q) .
\end{aligned}
$$

This transformation preserves equivalence under the (two-valued) completion semantics [11]. However, for stable or well-founded semantics, this is not the case. For instance, consider the $\mathcal{A L}$ theory $\mathcal{A}=\{P$ is caused if $Q ; Q$ is caused if $P\}$. In the original translation (1), neither $P$ nor $Q$ holds; in the second version (2), however, we obtain (ignoring the Head/2 and Prec/3 atoms for clarity):

$$
\begin{aligned}
\operatorname{Holds}(P) & \leftarrow \neg \operatorname{BodyNotSat}\left(R_{1}\right) . \\
\operatorname{BodyNotSat}\left(R_{1}\right) & \leftarrow \neg \operatorname{Holds}(Q) . \\
\operatorname{Holds}(Q) & \leftarrow \neg \operatorname{BodyNotSat}\left(R_{2}\right) . \\
\operatorname{BodyNotSat}\left(R_{2}\right) & \leftarrow \neg \operatorname{Holds}(P) .
\end{aligned}
$$

Under the stable semantics, this program has two models: $\{\operatorname{Holds}(P), \operatorname{Holds}(Q)\}$ and $\left\{\operatorname{BodyNotSat}\left(R_{1}\right), \operatorname{BodyNotSat}\left(R_{2}\right)\right\}$. As such, even though it might look reasonable at first, the Lloyd-Topor transformation does not preserve stable (or well-founded) models in this case.

Predicate introduction under the stable and well-founded semantics was considered by Van Gelder [14]. That paper, however, imposes strong restrictions on how newly introduced predicates can be defined. In particular, recursive definitions of such a new predicate are not allowed. However, the ability to introduce recursively defined new predicates can be very useful; indeed, it is precisely in this way that [1] manages to eliminate the universal quantifier in (1). Concretely, a predicate AllPrecHold $(r)$ is introduced to replace $\phi$ in (1), resulting in:

$$
\forall r, p \operatorname{Holds}(p) \leftarrow \operatorname{Head}(r, p) \wedge \operatorname{AllPrecHold}(r) .
$$

This predicate is then defined in terms of another new predicate, $\operatorname{AllFrom}(r, i)$, that means that the first $i$ preconditions of rule $r$ are satisfied. We then define this predicate by the following recursion:

$$
\begin{aligned}
& \forall r, n \operatorname{AllPrecHold}(r) \leftarrow \operatorname{AllFrom}(r, 1) . \\
& \forall r, n, q \operatorname{AllFrom}(r, n) \leftarrow \operatorname{Prec}(r, n, q) \wedge \operatorname{Holds}(q) \wedge \operatorname{AllFrom}(r, n+1) . \\
& \forall r, n, q \operatorname{AllFrom}(r, n) \leftarrow \operatorname{Prec}(r, n, q) \wedge \operatorname{Holds}(q) \wedge \operatorname{NbOfPrec}(r, n) .
\end{aligned}
$$


In this paper, we prove a generalization of Van Gelder's result, that shows that this translation is indeed equivalence preserving.

In the interest of easily proving this result in a general form, we will use the algebraic framework of approximation theory [3] (see also Section 2.1). This is a fixpoint theory for arbitrary (non-monotone) operators that generalizes all main semantics of various non-monotone logics, including Logic Programming, Default Logic and Autoepistemic Logic. As such, it allows properties of these different semantics for all of these logics to be studied in a uniform way. The central result of this paper (Section 3) is an abstract, algebraic fixpoint theorem, that will allow us to relate the stable and well-founded models of an original theory to those of the transformed theory. In Section 4, we instantiate this result to logic programming under the well-founded and stable model semantics, thereby generalizing the earlier result by Van Gelder [14]. In Section 5, we then discuss some applications of this result, including a general way of eliminating universal quantifiers by introducing recursively defined predicates.

In summary, the main contributions of this paper are the following:

- We prove a general, algebraic fixpoint result, that can be applied to Logic Programming, Default Logic, and Autoepistemic Logic.

- We apply this result to Logic Programming under the stable and wellfounded semantics, thus generalizing a result from [14]. In particular, our result also applies to recursively defined new predicates.

- We present a way of eliminating universal quantifiers from rule bodies through the introduction of recursively defined predicates. We use the result from the previous item to prove its correctness for stable and well-founded semantics. This offers an alternative for the corresponding step from the Lloyd-Topor transformation, which is only valid under completion semantics.

\section{Preliminaries}

In this section, we introduce some important concepts from approximation theory and show how these can be used to capture the stable and well-founded semantics for several Logic Programming variants.

\subsection{Approximation theory}

We use the following notations. Let $\langle L, \leq\rangle$ be a complete lattice. A fixpoint of an operator $O$ on $L$ is an element $x$ for which $x=O(x)$; a prefixpoint of $O$ is an $x$ such that $x \geq O(x)$. If $O$ is monotone, then it has a unique least fixpoint $x$, which is also its unique least prefixpoint. We denote this $x$ by $\operatorname{lfp}(O)$.

Our presentation of approximation theory is based on $[3,5]$. We consider the square $L^{2}$ of the domain of some lattice $L$. We will denote such an element as $\left(\begin{array}{ll}x & y\end{array}\right)$. We introduce the following projection functions: for a tuple $(x y)$, we denote by $[(x y) \mid$ the first element $x$ of this pair and by $\mid(x y)]$ the second element $y$. The obvious point-wise extension of $\leq$ to $L^{2}$ is called the product order on $L^{2}$, 
which we also denote by $\leq$ : i.e., for all $(x y),\left(x^{\prime} y^{\prime}\right) \in L^{2},(x y) \leq\left(x^{\prime} y^{\prime}\right)$ iff $x \leq x^{\prime}$ and $y \leq y^{\prime}$. An element $(x y)$ of $L^{2}$ can be seen as approximating certain elements of $L$, namely those in the (possibly empty) interval $[x, y]=\{z \in L \mid x \leq z$ and $z \leq y\}$. Using this intuition, we can derive a second order, the precision order $\leq_{p}$, on $L^{2}$ : for each $\left(\begin{array}{ll}x y \\ y\end{array}\right),\left(\begin{array}{ll}x^{\prime} & y^{\prime}\end{array}\right) \in L^{2},\left(\begin{array}{ll}x y & y\end{array} \leq_{p}\left(x^{\prime} y^{\prime}\right)\right.$ iff $x \leq x^{\prime}$ and $y^{\prime} \leq y$. Indeed, if $\left(\begin{array}{ll}x y & y\end{array} \leq_{p}\left(x^{\prime} y^{\prime}\right)\right.$, then $[x, y] \supseteq\left[x^{\prime}, y^{\prime}\right]$, i.e., $\left(x^{\prime} y^{\prime}\right)$ approximates fewer elements than $\left(\begin{array}{ll}x & y\end{array}\right)$. It can easily be seen that $\left\langle L^{2}, \leq_{p}\right\rangle$ is also a lattice. The structure $\left\langle L^{2}, \leq, \leq_{p}\right\rangle$ is the bilattice corresponding to $L$. If $\langle L, \leq\rangle$ is complete, then so are $\left\langle L^{2}, \leq\right\rangle$ and $\left\langle L^{2}, \leq_{p}\right\rangle$. Elements $(x x)$ of $L^{2}$ are called exact. The set of exact elements forms a natural embedding of $L$ in $L^{2}$.

Approximation theory is based on the study of operators which are monotone w.r.t. $\leq{ }_{p}$. Such operators are called approximations. An approximation $A$ approximates an operator $O$ on $L$ if for each $x \in L, A(x x)$ contains $O(x)$, i.e. $[A(x x)|\leq O(x) \leq| A(x x)]$. An exact approximation is one which maps exact elements to exact elements, i.e., for all $x \in L,\left[A\left(\begin{array}{ll}x & x\end{array}\right)|=| A\left(\begin{array}{ll}x & x)\end{array}\right)\right.$. Each exact approximation $A$ approximates a unique operator $O$ on $L$, namely the one that

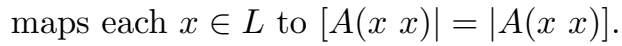

For an approximation $A$ on $L^{2}$, we define the operator $[A(\cdot y) \mid$ on $L$ that maps an element $x \in L$ to $[A(x y) \mid$, i.e. $[A(\cdot y) \mid=\lambda x$. $[A(x y) \mid$, and $\mid A(x \cdot)]$ that maps an element $y \in L$ to $\mid A(x y)]$. These operators are monotone. We define an operator $C_{A}^{\uparrow}$ on $L$, called the upward stable operator of $A$, as $C_{A}^{\uparrow}(y)=\operatorname{lfp}([A(\cdot y) \mid)$. We also define the downward stable operator $C_{A}^{\downarrow}$ of $A$ as $\left.C_{A}^{\downarrow}(x)=\operatorname{lfp}(\mid A(x \cdot)]\right)$. We define the stable operator $\mathcal{C}_{A}: L^{2} \mapsto L^{2}$ of $A$ by $\mathcal{C}_{A}(x y)=\left(C_{A}^{\uparrow}(y) C_{A}^{\downarrow}(x)\right)$. Because both $C_{A}^{\uparrow}$ and $C_{A}^{\downarrow}$ are anti-monotone, $\mathcal{C}_{A}$ is $\leq_{p}$-monotone.

An approximation $A$ defines a number of different fixpoints: the least fixpoint of an approximation $A$ is called its Kripke-Kleene fixpoint, fixpoints of its stable operator $\mathcal{C}_{A}$ are stable fixpoints and the least fixpoint of $\mathcal{C}_{A}$ is called the wellfounded fixpoint of $A$. As shown in $[3,5]$, these fixpoints correspond to various semantics of Logic Programming, Autoepistemic Logic and Default Logic.

\subsection{Rule sets and logic programming semantics}

We will try to prove our results in as general a setting as possible. To this end, we consider the following, quite general kind of rules: $\forall \boldsymbol{x} P(\boldsymbol{t}) \leftarrow \phi$. Here, $P$ is a predicate symbol, $\boldsymbol{x}$ is a tuple of variables, $\boldsymbol{t}$ is a tuple of terms, and $\phi$ is a first-order logic formula. For a rule $r$ of the above form, the atom $P(\boldsymbol{t})$ is called the head of $r$, while $\phi$ is known as its body.

We will consider the language consisting of sets of such rules, which we call rule sets. For such a rule set $\Delta$, we distinguish between two different kinds of predicates: a predicate that appears in the head of at least one rule of $\Delta$ is called a defined predicate; the other predicates are called open. We denote the set of all defined predicates by $\operatorname{Def}(\Delta)$ and that of all open ones by $O p(\Delta)$.

Syntactically, rule sets generalize a number of well-known Logic Programming variants. Normal logic programs are rule sets in which every body $\phi$ is a conjunction of literals. Standard Logic Programming semantics do not distin- 
guish between open and defined predicates. Rather, every predicate is treated as we will treat defined predicates. As such, we assume that for every open predicate $P$, there is a rule $\forall \boldsymbol{x} P(\boldsymbol{x}) \leftarrow$ false. In ID-logic [2,6], rule sets are used to represent inductive definitions of the defined predicates in terms of the open predicates. The distinction between open and defined predicates also appears in Answer Set Programming, where LP-functions are considered [10]. Such an LP-function is a rule set (which in [10] may also contain classical negation, unlike the rule sets we consider here) that specifies a mapping from interpretations of the open predicates to interpretations of the defined predicates. In deductive databases, rule sets also occur as definitions of intensional predicates.

We now define a class of semantics for such rule sets. For generality, we do this without restricting ourselves to the Herbrand universe. Our presentation is slightly non-standard, in the sense that we make no formal distinction between variables and constants; both will simply be called object symbols. An alphabet $\Sigma$ consists of a set of object symbols, function symbols, and predicate symbols. We interpret such an alphabet $\Sigma$ by a $\Sigma$-structure or $\Sigma$-interpretation. Such a $\Sigma$-interpretation $I$ consists of a domain $\operatorname{dom}(I)$, an interpretation of the object symbols of $\Sigma$ by domain elements, an interpretation of each function symbol $f / n$ of $\Sigma$ be an $n$-ary function on $D$, and an interpretation of each predicate symbol $P / n$ by an $n$-ary relation on $D$. If the alphabet $\Sigma$ is clear from the context, we often omit this from our notation. For any symbol $\sigma \in \Sigma$, we denote by $\sigma^{I}$ the interpretation of $\sigma$ by $I$. Similarly, for a term $t$ we denote the interpretation of $t$ by $t^{I}$ and we also extend this notation to tuples $\boldsymbol{t}$ of terms. For a structure $I$, an object symbol $x$, and a $d \in \operatorname{dom}(I)$, we denote by $I[x / d]$ the interpretation $J$ with the same domain as $I$, that interprets $x$ by $d$ and coincides with $I$ on everything else. We also extend this notation to tuples $\boldsymbol{x}$ and $\boldsymbol{d}$.

Most Logic Programming semantics make (at least implicit) use of the following way of evaluating formulas in pairs $(I J)$ of structures. The intuition here is that $I$ represents what is certainly true, while $J$ represents what is possibly true, i.e., everything that is false according to $J$ is certainly false. The following definition then tells us when a formula $\phi$ is certainly true in a pair $(I J)$.

Definition 1. Let $\phi$ be a formula. Let I and $J$ be structures, which coincide on their domain $D$ and their interpretation of both the object and function symbols. We now define when the pair $(I J)$ is a model of $\phi$, denoted $(I J) \models \phi$, by induction over the size of $\phi$ :

$-(I J) \models P(\boldsymbol{t})$ iff $I \models P(\boldsymbol{t})$, i.e., $\boldsymbol{t}^{I} \in P^{\boldsymbol{I}}$;

$-(I J) \models \neg \phi$ iff $(J I) \not \models \phi$;

- $(I J) \models \phi \vee \psi$ iff $(I J) \models \phi$ or $(I J) \models \psi$;

- $(I J) \models \exists x \phi(x)$ iff there exists a $d \in \operatorname{dom}(I)$, s.t. $(I[x / d] J[x / d]) \models \phi(x)$.

In the case for $\neg \phi$, the roles of $I$ and $J$ are switched. For a literal $\neg P(\boldsymbol{t})$, this means that $(I J) \models \neg P(\boldsymbol{t})$ iff $P(\boldsymbol{t})$ is false in $J$. If this is the case, then for all structures $K \leq J, K \models \neg P(\boldsymbol{t})$. For an atom $P(\boldsymbol{t})$, if $(I J) \models P(\boldsymbol{t})$, then for all structures $K \geq I, K \models P(\boldsymbol{t})$. By induction, it can now be shown that, for any $\phi$, if $(I J) \models \phi$, then for all structures $K \in[I, J]$, it is the case that $K \models \phi$. In 
this sense, $(I J) \models \phi$ holds when $\phi$ is certainly true. Let us now consider when $(J I) \models \phi$. For a literal $\neg P(\boldsymbol{t})$, this is the case iff $P(\boldsymbol{t})$ is false according to $I$. Whenever there exists a $K \geq I$ such that $K \models \neg P(\boldsymbol{t})$, this will be the case. For atom $P(\boldsymbol{t})$, if there exists a $K \leq J$ such that $K \models P(\boldsymbol{t})$, then $(J I) \models P(\boldsymbol{t})$. By induction, it can now be shown that, for any $\phi$, if $K \models \phi$ for some $K \in[I, J]$, then $(J I) \models \phi$. In this sense, $(J I) \models \phi$ holds when $\phi$ is possibly true. Hence, $(J I) \not \neq \phi$ when $\phi$ is certainly true.

This way of evaluating the truth of a formula $\phi$ has a strong connection to the standard four-valued Kleene truth assignment. In particular, we can define this four-valued valuation $\phi^{(I J)}$ as:

$\phi^{(I J)}=\mathbf{t}$ iff $(I J) \models \phi$ and $(J I) \models \phi ; \phi^{(I J)}=\mathbf{f}$ iff $(I J) \not \models \phi$ and $(J I) \not \models \phi$; $\phi^{(I J)}=\mathbf{u}$ iff $(I J) \not \models \phi$ and $(J I) \models \phi ; \phi^{(I J)}=\mathbf{i}$ iff $(I J) \models \phi$ and $(J I) \not \models \phi$.

A pre-interpretation for a rule set $\Delta$ over alphabet $\Sigma$ consists of an interpretation of the object symbols $\Sigma^{o}$ and function symbols $\Sigma^{f}$. Given a set of predicates $\boldsymbol{P}$, the class of all $\left(\Sigma^{o} \cup \Sigma^{f} \cup \boldsymbol{P}\right)$-structures that extend some fixed pre-interpretation $F$ is denoted as $L_{\boldsymbol{P}}^{F}$. We can define a truth order on this lattice as: $I \leq I^{\prime}$ iff $\forall P \in \boldsymbol{P}, P^{I} \subseteq P^{I^{\prime}}$. For this order, $L_{\boldsymbol{P}}^{F}$ is a complete lattice. Given a pair of interpretations for the open predicates $\left(O_{1} O_{2}\right)$ in $\left(L_{O p(\Delta)}^{F}\right)^{2}$, we will now define an immedeate consequence operator $\mathcal{T}_{\Delta}^{\left(O_{1} O_{2}\right)}$ on pairs of interpretations of the defined predicates, i.e., on $\left(L_{D e f(\Delta)}^{F}\right)^{2}$. This operator can be used to define a number of different Logic Programming semantics.

From now on, for a formula $\phi(\boldsymbol{x})$ and a tuple $\boldsymbol{d}$ of domain elements, we will write $(I J) \models \phi(\boldsymbol{d})$ instead of $(I[\boldsymbol{x} / \boldsymbol{d}] J[\boldsymbol{x} / \boldsymbol{d}]) \models \phi(\boldsymbol{x})$.

Definition 2. Let $\Delta$ be a rule set and $\left(O_{1}, O_{2}\right) \in\left(L_{O p(\Delta)}^{F}\right)^{2}$. We define a function $U_{\Delta}^{\left(O_{1} O_{2}\right)}$ from $\left(L_{D e f(\Delta)}^{F}\right)^{2}$ to $L_{D e f(\Delta)}^{F}$ as: $U_{\Delta}^{\left(O_{1} O_{2}\right)}(I J)=I^{\prime}$, where for each defined predicate $P / n: P^{I^{\prime}}$ consists of precisely those tuples $\boldsymbol{d} \in \operatorname{dom}(F)^{n}$, for which there exists a rule $(\forall \boldsymbol{x} P(\boldsymbol{t}) \leftarrow \phi(\boldsymbol{x})) \in \Delta$ and an $\boldsymbol{a} \in \operatorname{dom}(F)^{n}$, such that $\left(\left(O_{1} \cup I\right)\left(O_{2} \cup J\right)\right) \models \phi(\boldsymbol{a})$ and $\boldsymbol{t}^{F[\boldsymbol{x} / \boldsymbol{a}]}=\boldsymbol{d}$. We define the operator $\mathcal{T}_{\Delta}^{\left(O_{1} O_{2}\right)}$ on $\left(L_{D e f(\Delta)}^{F}\right)^{2}$ as $\mathcal{T}_{\Delta}^{\left(O_{1} O_{2}\right)}(I J)=\left(U_{\Delta}^{\left(O_{1} O_{2}\right)}(I J) U_{\Delta}^{\left(O_{2} O_{1}\right)}(J I)\right)$.

It can be shown that every $\mathcal{T}_{\Delta}^{\left(O_{1} O_{2}\right)}$ is an approximation. It approximates the well-known 2-valued immediate consequence operator $T_{\Delta}^{O}$, which can be defined as $T_{\Delta}^{O}(I)=\left[\mathcal{T}_{\Delta}^{(O O)}(I I) \mid\right.$. Because $\mathcal{T}_{\Delta}^{\left(O_{1} O_{2}\right)}$ is an approximation, it has a stable operator $\mathcal{C}_{\left.\mathcal{T}_{\Delta}^{\left(O_{1}\right.} O_{2}\right)}$. The well-founded model $\left(W_{1} W_{2}\right)$ of $\Delta$ given $\left(O_{1} O_{2}\right)$ is the least fixpoint of this stable operator. Similarly, a structure $S \in L_{D e f(\Delta)}^{F}$ is a stable model of $\Delta$ given $\left(O_{1} O_{2}\right)$ iff $S$ is a fixpoint of this stable operator.

A rule set $\Delta$ is monotone iff every $\mathcal{T}_{\Delta}^{\left(O_{1} O_{2}\right)}$ is a monotone operator (w.r.t. the product order $\leq$ ). For such rule sets, the well-founded model of $\Delta$ given some $\left(O_{1} O_{2}\right)$ can be shown to coincide with $\operatorname{lfp}\left(\mathcal{T}_{\Delta}^{\left(O_{1} O_{2}\right)}\right)$, which is also the unique stable model for $\Delta$ given $\left(O_{1} O_{2}\right)$. A rule set $\Delta$ is positive iff no defined predicate appears negatively in a rule body of $\Delta$. Such rule sets are always monotone.

We now introduce the following notions of the models of a rule set: 
Definition 3. Let $\Delta$ be a rule set and $F$ a pre-interpretation. Let $S_{1}, S_{2}, W_{1}, W_{2}$ be $\Sigma$-structures that extend $F$. The pair $\left(W_{1} W_{2}\right)$ is a model of $\Delta$ under the well-founded semantics, denoted $\left(W_{1} W_{2}\right) \models_{w} \Delta$ iff $\left.\left(W_{1} W_{2}\right)\right|_{D e f(\Delta)}$ is the wellfounded model of $\Delta$ under $\left.\left(W_{1} W_{2}\right)\right|_{O p(\Delta)}$. The pair $\left(S_{1} S_{2}\right)$ is a model of $\Delta$ under the stable model semantics, denoted $\left(S_{1} S_{2}\right) \models_{s} \Delta$ iff $\left.S_{1}\right|_{\text {Def }(\Delta)}=\left.S_{2}\right|_{\text {Def( }(\Delta)}$ and $\left.S_{1}\right|_{D e f(\Delta)}$ is a stable model of $\Delta$ under $\left.\left(S_{1} S_{2}\right)\right|_{O p(\Delta)}$.

For a normal logic program without open predicates, these semantics coincide with the well-known well-founded semantics [15] and stable model semantics [9]. Moreover, in ID-logic, a model of a definition $\Delta$ is defined as a structure $S$ for which $(S S) \models_{w} \Delta$. As such, by studying these semantics for rule sets, we will be able to derive results that are applicable to:

- Normal logic programs under stable and well-founded model semantics;

- LP-functions [10] (without classical negation);

- The well-founded semantics for logic programs with first-order bodies [14];

- Definitions in ID-logic [6].

\section{Fixpoint extension}

We want to study the following transformation. We start out with a rule set $\Delta$ in some alphabet $\Sigma$ and then introduce some new symbols $\Sigma_{n}$, e.g., the two predicates AllPrecHold and AllFrom from the example in the introduction. We then use these new predicates to form a new definition $\Delta^{\prime}$ over alphabet $\Sigma \cup \Sigma_{n}$. In order to study such transformations in an algebraic setting, we will assume two complete lattices $\left\langle L_{1}, \leq_{1}\right\rangle$ and $\left\langle L_{2}, \leq_{2}\right\rangle$. Here, $L_{1}$ can be thought of as consisting of the interpretations for the original alphabet $\Sigma$, while $L_{2}$ represents the interpretations for the additional new alphabet $\Sigma_{n}$. We will need to prove a result concerning the stable and well-founded models of $\Delta^{\prime}$, which means that we will need to work with pairs of interpretations of $\Sigma \cup \Sigma_{n}$. As such, in our algebraic setting, we consider the square $\left(L_{1} \times L_{2}\right)^{2}$ of the Cartesian product $L_{1} \times L_{2}$, which is isomorphic to the Cartesian product $L_{1}^{2} \times L_{2}^{2}$ of the

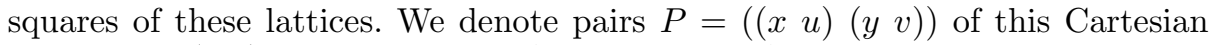
product by $\left(\begin{array}{ll}x & u \\ y & v\end{array}\right)$, where $(x u) \in L_{1}^{2}$ and $\left(\begin{array}{ll}y & v\end{array}\right) \in L_{2}^{2}$. We introduce the following projection functions: by $\left[P \mid\right.$ we denote the pair $\left(\begin{array}{l}x \\ y\end{array}\right)$, by $\left.\mid P\right]$ the pair $\left(\begin{array}{l}u \\ v\end{array}\right)$, by $\lceil P\rceil$ the pair $(x u)$, by $\lfloor P\rfloor$ the pair $(y v)$, by $\lfloor P \mid$ the element $y$, by $\lceil P \mid$ the element $x$, by $\mid P\rceil$ the element $u$, and by $\mid P\rfloor$ the element $v$.

Now, we want to prove a relation between the stable and well-founded fixpoints of the operator $\mathcal{T}_{\Delta}$ of the original definition $\Delta$ and those of the new operator $\mathcal{T}_{\Delta^{\prime}}$. Algebraically, we consider an approximation $A$ on the sqaure $L_{1}^{2}$ of the original lattice $L_{1}$ and an approximation $B$ on the extended lattice $L_{1}^{2} \times L_{2}^{2}$. We now impose some conditions to ensure a correspondence between the stable fixpoints of $A$ and $B$.

The main idea behind these conditions is the following. By introducing a new predicate into our original definition $\Delta$, we have added an additional "indirection". For instance, in the original version $\Delta$ of our example, we had the formula 
$\forall i, q \operatorname{Prec}(r, i, q) \Rightarrow \operatorname{Holds}(q)$, that could be evaluated in order to check whether all preconditons $q$ of rule $r$ were satisfied. This could be done by the $\mathcal{T}_{\Delta}$-operator in a single step. In our new definition $\Delta^{\prime}$, however, every application of $\mathcal{T}_{\Delta^{\prime}}$ only checks whether a single precondition is satisfied. Intuitively, to match the effect of a single application of $\mathcal{T}_{\Delta}$ to some pair $(X, Y)$ of interpretations of the alphabet of $\Delta$, we have to iterate $\mathcal{T}_{\Delta^{\prime}}$ long enough for the truth assignments of $(X, Y)$ to propagate throughout all of the new symbols of $\Delta^{\prime}$. Nevertheless, the end result of this iteration of $\mathcal{T}_{\Delta^{\prime}}$ should coincide with the result of the single application of $\mathcal{T}_{\Delta}$. We need some more notation to formalize this intuition.

Given the operator $B$ on $L_{1}^{2} \times L_{2}^{2}$ and a pair $(x u) \in L_{1}^{2}$, we define the operator

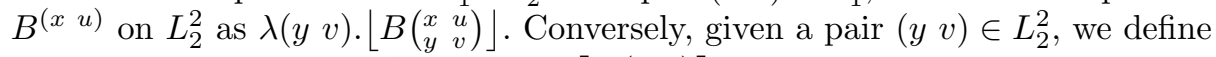

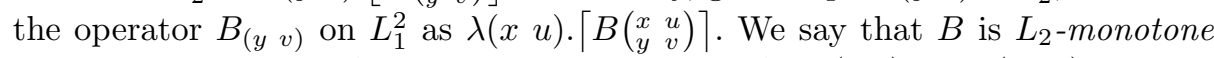
iff for each $(x \quad u) \in L_{1}^{2}$ and $(y v) \leq\left(\begin{array}{ll}y^{\prime} & v^{\prime}\end{array}\right) \in L_{2}^{2}, B\left(\begin{array}{ll}x & u \\ y & v\end{array}\right) \leq B\left(\begin{array}{ll}x & u \\ y^{\prime} & v^{\prime}\end{array}\right)$. If $B$ is $L_{2}$-monotone, then every operator $B^{(x u)}$ is monotone .

Definition 4 (Fixpoint extension). Let $B$ be an approximation on $L_{1}^{2} \times L_{2}^{2}$ and $A$ an approximation on $L_{1}^{2} . B$ is a fixpoint extension of $A$ iff

- B is $L_{2}$-monotone;

- For all $x, u \in L_{1}, B_{l f p\left(B^{(x u)}\right)}(x u)=A(x u)$.

The main algebraic result of this paper is that fixpoint extension preserves both stable and well-founded fixpoints.

Theorem 1 (Fixpoint extension). Let $B$ be a fixpoint extension of $A$. $\left(\begin{array}{ll}x & u \\ y & v\end{array}\right)$ is a fixpoint of the stable operator $\mathcal{C}_{B}$ iff $(x u)$ is a fixpoint of the stable operator $\mathcal{C}_{A}$ and $\left.(y v)=l f p\left(B^{(x} u\right)\right)$. Moreover, if $(x u)$ is the well-founded fixpoint of $A$ and $(y \quad v)=\operatorname{lfp}\left(B^{(x)} u\right)$, then the well-founded fixpoint of $B$ is precisely $\left(\begin{array}{ll}x & u \\ y & v\end{array}\right)$.

To prove this theorem, we first study some of the properties of $B$ and

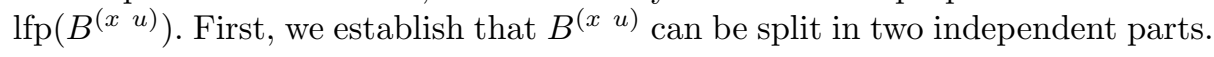

Lemma 1. Let $B$ be $L_{2}$-monotone. Then for all $y, v \in L_{2}, B^{(x u)}(y v)=$ $\left(B_{1}^{(x u)}(y) B_{2}^{(x u))}(v)\right)$, where the operators $B_{1}^{(x u)}$ and $B_{2}^{(x u)}$ on $L_{2}$ are defined as $B_{1}^{(x u)}(y)=\left[B^{(x u)}(y \top) \mid\right.$ and $\left.B_{2}^{(x u)}(v)=\mid B^{(x u)}(\top v)\right]$.

Proof. For $B_{1}^{(x u)}$, it suffices to show that, for all $y, v \in L_{2}, B^{(x u)}(y v)$ and

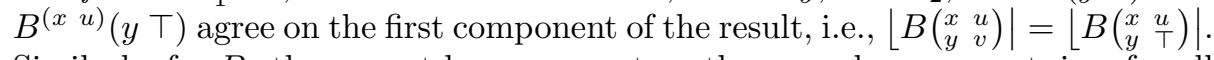
Similarly, for $B_{2}$ there must be agreement on the second component, i.e., for all $\left.\left.y, v \in L_{2}: \mid B\left(\begin{array}{ll}x & u \\ y & v\end{array}\right)\right\rfloor=\mid B\left(\begin{array}{ll}x & u \\ \top & v\end{array}\right)\right\rfloor$. We will only prove the equality for $\left.B_{1}^{(x} u\right)$; the proof of the second one is analogous.

First, we note that $\left(\begin{array}{ll}x & u \\ y & v\end{array}\right) \geq_{p}\left(\begin{array}{ll}x & u \\ y & T\end{array}\right)$. By $\leq_{p}$-monotonicity of $B$, this implies that $\left|B\left(\begin{array}{ll}x & u \\ y & v\end{array}\right)\right| \geq\left|B\left(\begin{array}{ll}x & u \\ y & \mathrm{~T}\end{array}\right)\right|$. Secondly, we also note that $\left(\begin{array}{ll}x & u \\ y & v\end{array}\right) \leq\left(\begin{array}{ll}x & u \\ y & \mathrm{~T}\end{array}\right)$. By $L_{2^{-}}$ monotonicity of $B$, this implies that $\left\lfloor B\left(\begin{array}{ll}x & u \\ y & v\end{array}\right) \mid \leq\left\lfloor B\left(\begin{array}{ll}x & u \\ y & T\end{array}\right) \mid\right.\right.$. Combining these two inequalities gives the desired result. 
The stable operator $\mathcal{C}_{B}$ of an approximation $B$ is defined in terms of its downward and upward stable operators $C_{B}^{\downarrow}$ and $C_{B}^{\uparrow}$. We show the following relation between these operators and the operators $B_{1}^{(x u)}$ and $B_{2}^{(x u)}$ from Lemma 1.

Lemma 2. If $\left(\begin{array}{l}x \\ y\end{array}\right)=C_{B}^{\uparrow}\left(\begin{array}{l}u \\ v\end{array}\right)$, then $y=\operatorname{lfp}\left(B_{1}^{(x)} u\right)$. If $\left(\begin{array}{l}u \\ v\end{array}\right)=C_{B}^{\downarrow}\left(\begin{array}{l}x \\ y\end{array}\right)$, then $v=$ $\operatorname{lfp}\left(B_{2}^{(x u)}\right)$.

Proof. We only prove the first implication; the proof of the second one is analogous. Let $z=\operatorname{lfp}\left(B_{1}^{(x u)}\right)$. We will show that $y=z$. We start by showing that $z \leq y$. By definition of $C_{B},\left(\begin{array}{l}x \\ y\end{array}\right)=\left[B\left(\begin{array}{ll}x & u \\ y & v\end{array}\right) \mid\right.$. In particular, $y=B_{1}^{(x)} u(y)$, i.e., $y$ is a fixpoint of $B_{1}^{(x u)}$. Because $z$ was chosen to be the least fixpoint of this operator, $z \leq y$. Now, we prove that $y \leq z$. Because $z \leq y$, it is the case that $\left(\begin{array}{ll}x & u \\ z & v\end{array}\right) \leq\left(\begin{array}{ll}x & u \\ y & v\end{array}\right)$. By $L_{2}$-monotonicity of $B$, this implies that $B\left(\begin{array}{ll}x & u \\ z & v\end{array}\right) \leq B\left(\begin{array}{ll}x & u \\ y & v\end{array}\right)$ and, in particular, $\left\lceil B\left(\begin{array}{ll}x & u \\ z & v\end{array}\right) \mid \leq\left\lceil B\left(\begin{array}{ll}x & u \\ y & v\end{array}\right) \mid=x\right.\right.$. Because $z$ is a fixpoint of $\left.B_{1}^{(x} u\right)$, we also have that $\left|B\left(\begin{array}{ll}x & u \\ z & v\end{array}\right)\right|=z$. As such, $\left[B\left(\begin{array}{ll}x & u \\ z & v\end{array}\right) \mid \leq\left(\begin{array}{l}x \\ z\end{array}\right)\right.$ or, in other words, $\left(\begin{array}{l}x \\ z\end{array}\right)$ is a prefixpoint of $\left[B\left(\begin{array}{ll}u \\ :\end{array}\right) \mid\right.$. Because the least fixpoint $\left(\begin{array}{l}x \\ y\end{array}\right)$ of this operator is also its least prefixpoint, $\left(\begin{array}{l}x \\ y\end{array}\right) \leq\left(\begin{array}{l}x \\ z\end{array}\right)$; in particular, $y \leq z$.

In order to prove the correspondence between well-founded fixpoints, we will also need to take the precision order into account.

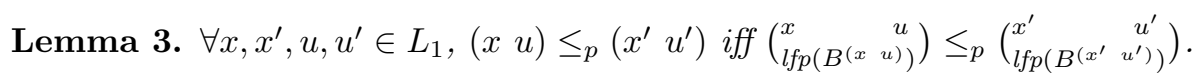

Proof. It is clear that the right hand side of this equivalence directly implies the left. Let $x, x^{\prime}, u, u^{\prime}$ be as above and let $(y v)=\operatorname{lfp}\left(B^{(x u)}\right)$ and $\left(y^{\prime} v^{\prime}\right)=$ $\operatorname{lfp}\left(B^{\left(x^{\prime} u^{\prime}\right)}\right)$. It suffices to show that $(y v) \leq_{p}\left(y^{\prime} v^{\prime}\right)$. We first show that $y \leq y^{\prime}$. By Lemma 1, $y=\operatorname{lfp}\left(B_{1}^{(x u)}\right)$. Because this implies that $y$ is also the least prefixpoint of $B_{1}^{(x u)}$, it now suffices to show that $y^{\prime}$ is a prefixpoint of $B_{1}^{(x u)}$ as well, i.e., that $\left.y^{\prime} \geq B_{1}^{(x} u\right)\left(y^{\prime}\right)$. Because for any $w,\left(\begin{array}{ll}x^{\prime} & u^{\prime} \\ y^{\prime} & w\end{array}\right) \geq_{p}\left(\begin{array}{ll}x & u \\ y^{\prime} & w\end{array}\right)$, we have that $y^{\prime}=\left\lfloor B\left(\begin{array}{ll}x^{\prime} & u^{\prime} \\ y^{\prime} & w\end{array}\right) \mid \geq\left\lfloor B\left(\begin{array}{ll}x & u \\ y^{\prime} & w\end{array}\right) \mid=B_{1}^{(x} u\left(y^{\prime}\right)\right.\right.$.

We now show that $v \geq v^{\prime}$. By Lemma $1, v^{\prime}=\operatorname{lfp}\left(B_{2}^{\left(x^{\prime} u^{\prime}\right)}\right)$. This implies that $v^{\prime}$ is also the least prefixpoint of $B_{2}^{\left(x^{\prime} u^{\prime}\right)}$ and, therefore, it suffices to show that $v \geq B_{2}^{\left(x^{\prime} u^{\prime}\right)}(v)$. Because for any $z,\left(\begin{array}{ll}x^{\prime} & u^{\prime} \\ z & v\end{array}\right) \geq_{p}\left(\begin{array}{ll}x & u \\ z & v\end{array}\right)$, we have that: $\left.B_{2}^{\left(x^{\prime}\right.} u^{\prime}\right)(v)=$ $\left.\left.\mid B\left(\begin{array}{ll}x^{\prime} & u^{\prime} \\ z & v\end{array}\right)\right\rfloor \leq \mid B\left(\begin{array}{ll}x & u \\ z & v\end{array}\right)\right\rfloor=v$.

Proof (of Theorem 1).

We first show that if $\left(\begin{array}{ll}x & u \\ y & v\end{array}\right)$ is a fixpoint of $\mathcal{C}_{B}$, then $\left(\begin{array}{ll}x & u\end{array}\right)$ is a fixpoint of $\mathcal{C}_{A}$

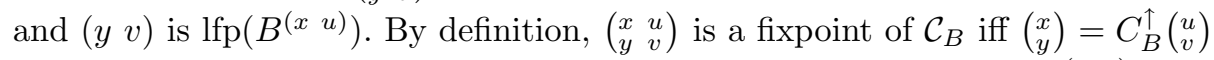
and $\left(\begin{array}{l}u \\ v\end{array}\right)=C_{B}^{\downarrow}\left(\begin{array}{l}x \\ y\end{array}\right)$. By Lemma 2, if this is the case, then $y=\operatorname{lfp}\left(B_{1}^{(x u)}\right)$ and $v=\operatorname{lfp}\left(B_{2}^{(u x)}\right)$. As such $(y v)=\operatorname{lfp}\left(B^{(x u)}\right)$. Because $B$ is an extension of $A$, we now have that $\left\lceil B\left(\begin{array}{ll}x & u \\ y & v\end{array}\right)\right\rceil=A(x)$.

To prove the other direction, let $(x u)=\mathcal{C}_{A}(x u)$ and let $(y v)$ be $\operatorname{lfp}\left(B^{(x u)}\right)$. We need to show that $\left(\begin{array}{ll}x & u \\ y & v\end{array}\right)$ is a fixpoint of $\mathcal{C}_{B}$, i.e., that $\left(\begin{array}{l}x \\ y\end{array}\right)=C_{B}^{\uparrow}\left(\begin{array}{l}u \\ v\end{array}\right)$ and 
$\left(\begin{array}{l}u \\ v\end{array}\right)=C_{B}^{\downarrow}\left(\begin{array}{l}x \\ y\end{array}\right)$. We only show that $\left(\begin{array}{l}x \\ y\end{array}\right)=C_{B}^{\uparrow}\left(\begin{array}{l}u \\ v\end{array}\right)$; the proof of the other equality is

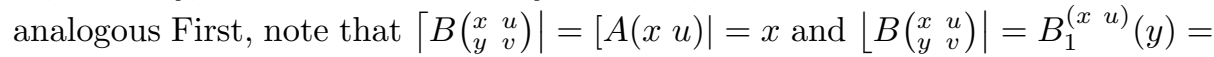
$y$. Hence, $\left[B\left(\begin{array}{ll}x & u \\ y & v\end{array}\right) \mid=\left(\begin{array}{l}x \\ y\end{array}\right)\right.$, i.e., $\left(\begin{array}{l}x \\ y\end{array}\right)$ is a fixpoint of $\left[B\left(\begin{array}{l}u \\ v\end{array}\right) \mid\right.$. It now suffices to show that $\left(\begin{array}{l}x \\ y\end{array}\right)$ is the least such fixpoint. Assume that $\left(\begin{array}{l}a \\ b\end{array}\right)$ is such that $\left[B\left(\begin{array}{ll}a & u \\ b & v\end{array}\right) \mid=\left(\begin{array}{l}a \\ b\end{array}\right)\right.$ and $\left(\begin{array}{l}a \\ b\end{array}\right) \leq\left(\begin{array}{l}x \\ y\end{array}\right)$. We show that $\left(\begin{array}{l}a \\ b\end{array}\right) \geq\left(\begin{array}{l}x \\ y\end{array}\right)$. Let $\left(b^{\prime} c\right)=\operatorname{lfp}\left(B^{(a} u\right)$. Because $b$ is a fixpoint of $\left.B_{1}^{(a} u\right), b^{\prime} \leq b$ and therefore $\left(\begin{array}{ll}a & u \\ b^{\prime} & c\end{array}\right) \leq_{p}\left(\begin{array}{ll}a & u \\ b & c\end{array}\right)$.

By Lemma 3, the fact that $a \leq x$ implies that $c \geq v$. Consequently, we have that $\left(\begin{array}{ll}a & u \\ b & c\end{array}\right) \leq_{p}\left(\begin{array}{ll}a & u \\ b & v\end{array}\right)$ and, therefore, by $\leq{ }_{p}$-monotonicity of $B$ :

$$
\left[A\left(\begin{array}{ll}
a & u
\end{array}\right) \mid=\left\lceil B\left(\begin{array}{cc}
a & u \\
b^{\prime} & c
\end{array}\right) \mid \leq\left\lceil B\left(\begin{array}{ll}
a & u \\
b & c
\end{array}\right) \mid \leq\left\lceil B\left(\begin{array}{ll}
a & u \\
b & v
\end{array}\right) \mid=a\right.\right.\right.\right.
$$

Hence, $a$ is a prefixpoint of $[A(\cdot u) \mid$. Because $x$ is the least such prefixpoint, $x \leq a$ and, therefore, $x=a$. By Lemma 2, this also implies that $b=y$.

The fact that $\left(\begin{array}{l}u \\ v\end{array}\right)=C_{B}^{\downarrow}\left(\begin{array}{l}x \\ y\end{array}\right)$ can be shown in the same way.

Given this one-to-one correspondence between stable fixpoints of $A$ and those of $B$, the correspondence between the well-founded fixpoint of $A$ and that of $B$ follows directly from Lemma 3 .

\section{Application to rule sets}

In this section, we use the algebraic results of Section 3 to derive a concrete equivalence theorem for rule sets. Recall that we are interested in transformations from some original rule set $\Delta$ over an alphabet $\Sigma$ into a new rule set $\Delta^{\prime}$ over an alphabet $\Sigma^{\prime} \supseteq \Sigma$. More concretely, $\Delta^{\prime}$ is the result of replacing a subformula $\phi(\boldsymbol{x})$ of some rule of $\Delta$ by a new predicate $P(\boldsymbol{x})$ and adding a new rule set $\delta$ to $\Delta$ to define this new predicate $P$. We will denote the result of replacing (some fixed set of occurrences of) $\phi(\boldsymbol{x})$ in $\Delta$ by $P(\boldsymbol{x})$ as $\Delta[\phi(\boldsymbol{x}) / P(\boldsymbol{x})]$, i.e., $\Delta^{\prime}=\Delta[\phi(\boldsymbol{x}) / P(\boldsymbol{x})] \cup \delta$. We will assume that $\Sigma \cap \operatorname{Def}(\delta)$ is empty.

We now impose some criteria to ensure that, for certain interpretations $O$ of the open predicates of $\Delta^{\prime}$, the operator $\mathcal{T}_{\Delta^{\prime}}^{(O)}$ is a fixpoint extension of $\mathcal{T}_{\Delta}^{(O O)}$. We state our theorem for the set $C$ of all structures that extend $O$ to $\Sigma^{\prime}$.

Theorem 2 (Predicate introduction). Let $\Delta$ be a rule set and let $\Delta^{\prime}$ be the result of replacing some positive occurences of $\phi(\boldsymbol{x})$ by $P(\boldsymbol{x})$ defined in $\delta$, as outlined above. If the following conditions are satisfied:

1. $\delta$ is a monotone rule set;

2. for all $I, J \in C$ s.t. $(I J) \models_{s} \delta: \forall \boldsymbol{a} \in D^{n}, P(\boldsymbol{a})^{(I J)}=\phi(\boldsymbol{a})^{(I J)}$;

then for all $I, J$ in $C,\left.(I I)\right|_{\Sigma} \models_{s} \Delta$ iff $(I I) \models_{s} \Delta^{\prime}$ and $\left.(I J)\right|_{\Sigma} \models_{w} \Delta$ iff $(I J) \models_{w} \Delta^{\prime}$.

Observe that because $\delta$ is monotone, we could have equivalently used $\models_{w}$ instead of $\models_{s}$. To see that this theorem applies to our example from the introduction, let $\delta$ be the rules given in (5). Clearly, $\delta$ is positive. Now, if we restrict 
our attention to those interpretations $O$ for $\operatorname{Open}\left(\Delta^{\prime}\right)$ that actually correspond to $\mathcal{A L}$-rules ${ }^{1}$, then it is easy to see that for all $r \in \operatorname{dom}(O), \operatorname{AllPrecHold}(r)^{(I J)}$ iff $\phi(r)^{(I J)}$. As such, Condition 2 is satisfied.

Proof (of Theorem 2). Let $L=\left\{\left.I\right|_{\operatorname{Def}(\Delta)} \mid I \in C\right\}$ and $L^{\prime}=\left\{\left.I\right|_{\operatorname{Def}(\delta)} \mid I \in C\right\}$. Now, $C$ is isomorphic to $L \times L^{\prime}$. Let $\mathcal{T}$ be $\mathcal{T}_{\Delta}^{(O O)}: L^{2} \rightarrow L^{2}$ and $\mathcal{T}^{\prime}$ be $\mathcal{T}_{\Delta^{\prime}}^{(O)}{ }^{(O)}$ : $\left(L \times L^{\prime}\right)^{2} \rightarrow\left(L \times L^{\prime}\right)^{2}$. It suffices to prove that $\mathcal{T}^{\prime}$ is a fixpoint extension of $\mathcal{T}$, i.e., that (1) $\mathcal{T}^{\prime}$ is $L^{\prime}$-monotone and $(2)\left\lceil\mathcal{T}_{\operatorname{lfp}\left(\mathcal{T}^{\prime(I J)}\right)}^{\prime}(I J)\right\rceil=\mathcal{T}(I J)$ for any $I, J \in L$.

We first show (1). Let $(I J) \in L^{2}$ and $\left(I_{1}^{\prime} J_{1}^{\prime}\right) \leq\left(I_{2}^{\prime} J_{2}^{\prime}\right) \in L^{\prime 2}$. We have to prove the inequality $\mathcal{T}^{\prime}\left(\begin{array}{ll}I & J \\ I_{1}^{\prime} & J_{1}^{\prime}\end{array}\right) \leq \mathcal{T}^{\prime}\left(\begin{array}{ll}I & J \\ I_{2}^{\prime} & J_{2}^{\prime}\end{array}\right)$. By the monotonicity of $\delta$, we

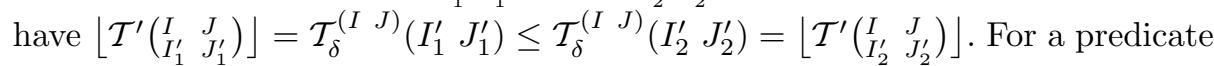
$Q \in \operatorname{Def}(\Delta)$ and domain elements $\boldsymbol{a}$, s.t. $\left[\mathcal{T}^{\prime}\left(\begin{array}{cc}I & J_{1}^{\prime} \\ I_{1}^{\prime} & J_{1}^{\prime}\end{array}\right) \mid \models Q(\boldsymbol{a})\right.$, there exist a rule $\forall \boldsymbol{x} Q(\boldsymbol{t}) \leftarrow \psi(\boldsymbol{x})$ and domain elements $\boldsymbol{d}$ such that $\boldsymbol{t}^{O[\boldsymbol{x} / \boldsymbol{d}]}=\boldsymbol{a}$. Then $\left(\begin{array}{cc}I & J \\ I_{1}^{\prime} & J_{1}^{\prime}\end{array}\right)=\psi(\boldsymbol{d})$. Since $P$ is the only predicate of $\operatorname{Def}(\delta)$ that can occur in $\psi$ and because it can only occur positively, this implies $\left(\begin{array}{ll}I & J_{2}^{\prime} \\ I_{2}^{\prime} & J_{2}^{\prime}\end{array}\right) \models \psi(\boldsymbol{d})$. Hence, also in this case $Q(\boldsymbol{a}) \in\left[\mathcal{T}^{\prime}\left(\begin{array}{cc}I & J \\ I_{2}^{\prime} & J_{2}^{\prime}\end{array}\right) \mid\right.$. For $Q(\boldsymbol{a})$ such that $\left.\mid \mathcal{T}^{\prime}\left(\begin{array}{cc}I & J \\ I_{1}^{\prime} & J_{1}^{\prime}\end{array}\right)\right] \models Q(\boldsymbol{a})$, an analogous proof can be given to complete our proof of $L^{\prime}$-monotonicity.

We now show $(2)$. Let $\left(I^{\prime} J^{\prime}\right)=\operatorname{lfp}\left(\mathcal{T}^{\prime(I J)}\right)$. Then $\left(I^{\prime} J^{\prime}\right)=\operatorname{lfp}\left(\mathcal{T}_{\delta}^{(I \cup O J \cup O)}\right)$, and, therefore, by the monotonicity of $\delta,\left(\begin{array}{ll}I & J \\ I^{\prime} & J^{\prime}\end{array}\right) \models_{s} \delta$. Denoting $I_{2}=\left(\begin{array}{l}I \\ I^{\prime}\end{array}\right)$ and $J_{2}=\left(\begin{array}{l}J \\ J^{\prime}\end{array}\right)$, we then have by condition 2 that $\left.\forall \boldsymbol{c} \in D^{n} P(\boldsymbol{c})^{\left(I_{2} J_{2}\right)}=\phi(\boldsymbol{c})^{\left(I_{2}\right.} J_{2}\right)$, hence $\left(I_{2} J_{2}\right) \models P(\boldsymbol{c})$ iff $\left(I_{2} J_{2}\right) \models \phi(\boldsymbol{c})$.

We also have that $[\mathcal{T}(I J) \mid \models Q(\boldsymbol{a})$ iff $\Delta$ contains a rule $\forall \boldsymbol{x} Q(\boldsymbol{t}) \leftarrow \psi(\boldsymbol{x})$ and domain elements $\boldsymbol{d}$ exist such that $\boldsymbol{t}^{O[\boldsymbol{x} / \boldsymbol{d}]}=\boldsymbol{a}$ and $(I J) \models \psi(\boldsymbol{d})$. The corresponding rule in $\Delta^{\prime}$ is $\forall \boldsymbol{x} Q(\boldsymbol{t}) \leftarrow \psi^{\prime}(\boldsymbol{x})$. Consequently, $\left(I_{2} J_{2}\right) \models \psi(\boldsymbol{d})$ iff $\left(\begin{array}{ll}I_{2} & J_{2}\end{array}\right) \models \psi^{\prime}(\boldsymbol{d})$. The proof for a $Q(\boldsymbol{a})$ such that $\left.\mid \mathcal{T}(I J)\right] \models Q(\boldsymbol{a})$ is analogous.

Condition 2 of Theorem 2 requires us to check a four-valued equivalence between $P(\boldsymbol{x})$ as defined by $\delta$ and the original formula $\phi(\boldsymbol{x})$. One might wonder whether this is really necessary, i.e., whether it would suffice to check only the following two-valued equivalence:

$$
\text { For all } I \in C \text { s.t. }(I I) \models{ }_{s} \delta: \forall \boldsymbol{a} \in D^{n}, I \models P(\boldsymbol{a}) \text { iff } I \models \phi(\boldsymbol{a}) \text {. }
$$

In general, this is not the case. For instance, consider an attempt to replace in $\Delta=\{R \leftarrow Q \vee \neg Q$. $\}$ the formula $\phi=Q \vee \neg Q$ by a new predicate $P$, defined by a definition $\delta=\{P$. $\}$. In this case, the above equivalence and all other conditions of Theorem 2 would be satisfied, but the well-founded model of $\Delta^{\prime}=\{R \leftarrow P$.P. $\}$ is $(\{R, P\}\{R, P\})$, while that of $\Delta$ is $(\{\}\{R, P\})$.

The four-valued way of interpreting formulas is an integral part of both stable and well-founded semantics. Therefore, it makes sense that, as the above example

\footnotetext{
${ }^{1}$ More specifically, for every $r$ and $i$ there should a unique $q$ such that $(r, i, q) \in \operatorname{Prec}^{O}$ holds. Moreover, if for some $r$ there are $n$ such $i$ 's, then $n$ should be the unique $m$ for which $(r, m) \in N b O f \operatorname{Prec}^{O}$.
} 
shows, a four-valued equivalence is required in order to preserve either of these semantics. In practice, however, this should not pose too much of a problem, since most common transformations from classical logic, e.g. the De Morgan laws, are still equivalence preserving in the four-valued case.

\section{Applications and Related Work}

The kind of transformations considered in this paper have a long history in Logic Programming. In particular, we consider three related investigations:

- Lloyd and Topor [11] introduced transformations that preserve equivalence under the 2-valued completion semantics. As shown in our example in Section 1, these transformations do not preserve equivalence under the wellfounded or stable model semantics. As such, our result can be seen as an attempt to provide Lloyd-Topor-like transformations for these semantics.

- Van Gelder [14] presented a logic of alternating fixpoints to generalize the well-founded semantics to arbitrary rule bodies. In the same paper, he established the result given below as Theorem 3. We discuss the relation of our work with this result in Section 5.1

- The "Principle of Partial Evaluation" (PPE) was introduced by Dix in a study of properties for classifying logic programming semantics [7]. As we discuss in Section 5.2, this principle has a strong relation with our work.

More recently, there has been a lot of work in Answer Set Programming on the topic of strong equivalence. In general, the transformations we considered here do not preserve strong equivalence, because they are specific to a particular rule set.

\subsection{Predicate extraction and eliminating $\forall$}

The following result by Van Gelder dates back to 1993:

Theorem 3 ([14]). Let $\Delta$ be a rule set containing a rule $r=\forall \boldsymbol{x} P(\boldsymbol{t}) \leftarrow \psi$. Let $\phi(\boldsymbol{y})$ be an existentially quantified conjunction of literals, and let $Q$ be a new predicate symbol. If $\phi(\boldsymbol{y})$ is a positive subformula of $\psi$, then $\Delta$ is equivalent under the stable and well-founded semantics to the rule set $\Delta^{\prime}$, that results from replacing $\phi(\boldsymbol{y})$ in $r$ by $Q(\boldsymbol{y})$ and adding the rule $\forall \boldsymbol{y} Q(\boldsymbol{y}) \leftarrow \phi(\boldsymbol{y})$ to $\Delta$.

Because the rule set $\delta=\{\forall \boldsymbol{y} Q(\boldsymbol{y}) \leftarrow \phi(\boldsymbol{y})$. $\}$ clearly satisfies the conditions of Theorem 2, Van Gelder's theorem follows directly from ours. This result provides a theoretical justification for the common programming practice of predicate extraction: replacing a subformula that occurs in multiple rules by a new predicate to make the program more concise and more readable. In [13], predicate extraction is considered to be an important refactoring operation (i.e., an equivalence preserving transformation to improve maintainability) for Logic Programming.

Our result extends Van Gelder's theorem by allowing the new predicate $Q$ to be defined by an additional rule set $\delta$, instead of allowing only the definition 
$\{\forall \boldsymbol{y} Q(\boldsymbol{y}) \leftarrow \phi(\boldsymbol{y})$.$\} . In particular, recursive definitions of Q$ are also allowed. This significantly increases the applicability of the theorem. Indeed, as we already illustrated in the introduction, it allows us to eliminate certain universial quantifiers. The general idea behind this method is that we can replace a universal quantifier by a recursion over some total order on the domain.

Definition 5 (Domain iterator). Let $C$ be a set of $\Sigma$-structures with domain D. Let First/1, Next/2 and Last/1 be predicate symbols of $\Sigma$. The triple $\langle$ First, Next, Last is a domain iterator in $C$ iff for each structure $S \in C$ : Next ${ }^{S}$ is a total order on D, with a minimal element $f$ and a maximal element $l$ such that First ${ }^{S}=\{f\}$ and Last $^{S}=\{l\}$.

Given such a domain iterator $I t=\langle$ First, Next, Last $\rangle$, we can introduce the following rule set $\delta_{\phi}^{I t}$ to define a new predicate $\operatorname{Forall}(\boldsymbol{x})$ as a replacement for some $\phi(\boldsymbol{x})=\forall y \psi(\boldsymbol{x}, y)$ :

$$
\begin{aligned}
\forall \boldsymbol{x}, y \operatorname{Forall}(\boldsymbol{x}) & \leftarrow \operatorname{First}(y) \wedge \operatorname{AllFrom}(\boldsymbol{x}, y) . \\
\forall \boldsymbol{x}, y \operatorname{AllFrom}(\boldsymbol{x}, y) & \leftarrow \psi(\boldsymbol{x}, y) \wedge \operatorname{Next}\left(y, y^{\prime}\right) \wedge \operatorname{AllFrom}\left(\boldsymbol{x}, y^{\prime}\right) . \\
\forall \boldsymbol{x}, y \operatorname{AllFrom}(\boldsymbol{x}, y) & \leftarrow \psi(\boldsymbol{x}, y) \wedge \operatorname{Last}(y) .
\end{aligned}
$$

The following result now follows from Theorem 2. Due to space limitations, we omit a formal proof.

Theorem 4 ( $\forall$ elimination). Let $\Delta$ be a rule set and $\phi(\boldsymbol{x})$ a positive subformula of the form $\forall y \psi(\boldsymbol{x}, y)$. For a set of structures $C$ with finite domain, if It is a domain iterator, then $\Delta[\phi /$ Forall $] \cup \delta_{\phi}^{I t}$ is equivalent to $\Delta$ under stable and well-founded semantics.

In this theorem, we assume a total order on the entire domain and this same order can be used to eliminate all universally quantified formulas, that satisfy the condition of the theorem. This is not precisely what happened in our example. Indeed, there, the universally quantified formula $\phi(\boldsymbol{x})$ was of the form: $\forall \boldsymbol{y} \Psi_{1}(\boldsymbol{x}, \boldsymbol{y}) \Rightarrow \Psi_{2}(\boldsymbol{x}, \boldsymbol{y})$. Using the above theorem, we would replace $\phi(\boldsymbol{x})$ by a recursion that says that the implication $\Psi_{1}(\boldsymbol{x}) \Rightarrow \Psi_{2}(\boldsymbol{x})$ must hold for every element in the domain. However, in our original version of this example, we actually replaced $\phi(\boldsymbol{x})$ by a recursion which says that for all $\boldsymbol{y}$ that satisfy $\Psi_{1}(\boldsymbol{x}, \boldsymbol{y})$ (i.e., for all $i, q$ s.t. $\left.\operatorname{Prec}(r, i, q)\right)$ the consequent $\Psi_{2}(\boldsymbol{x}, \boldsymbol{y})$ (i.e., Holds $(q))$ is satisfied. This is a slightly different approach, which we can also prove in general. A restricted iterator for $\boldsymbol{y}$ in $\psi_{1}(\boldsymbol{x}, \boldsymbol{y})$ is a triple of predicates $\left\langle F i r s t(\boldsymbol{x}, \boldsymbol{y}), N \operatorname{ext}\left(\boldsymbol{x}, \boldsymbol{y}, \boldsymbol{y}^{\prime}\right), \operatorname{Last}(\boldsymbol{x}, \boldsymbol{y})\right\rangle$, s.t. for every tuple $\boldsymbol{d}$ of domain elements, $\left\langle F \operatorname{First}(\boldsymbol{d}, \boldsymbol{y}), N \operatorname{ext}\left(\boldsymbol{d}, \boldsymbol{y}, \boldsymbol{y}^{\prime}\right), \operatorname{Last}(\boldsymbol{d}, \boldsymbol{y})\right\rangle$ is an iterator over $\left\{\boldsymbol{e} \in D^{n}\right.$ $\left.\Psi_{1}(\boldsymbol{d}, \boldsymbol{e})\right\}$.

Given such a restricted iterator, we can define the following replacement $\operatorname{Forall}(\boldsymbol{x})$ for $\phi(\boldsymbol{x})$ :

$$
\begin{aligned}
\forall \boldsymbol{x}, \boldsymbol{y} \operatorname{Forall}(\boldsymbol{x}) & \leftarrow \operatorname{First}(\boldsymbol{x}, \boldsymbol{y}) \wedge \operatorname{AllFrom}(\boldsymbol{x}, \boldsymbol{y}) . \\
\forall \boldsymbol{x}, \boldsymbol{y}, \boldsymbol{y}^{\prime} \operatorname{AllFrom}(\boldsymbol{x}, \boldsymbol{y}) & \leftarrow \Psi_{2}(\boldsymbol{x}, \boldsymbol{y}) \wedge \operatorname{Next}\left(\boldsymbol{x}, \boldsymbol{y}, \boldsymbol{y}^{\prime}\right) \wedge \operatorname{AllFrom}\left(\boldsymbol{x}, \boldsymbol{y}^{\prime}\right) . \\
\forall \boldsymbol{x}, \boldsymbol{y} \operatorname{AllFrom}(\boldsymbol{x}, \boldsymbol{y}) & \leftarrow \Psi_{2}(\boldsymbol{x}, \boldsymbol{y}) \wedge \operatorname{Last}(\boldsymbol{x}, \boldsymbol{y}) .
\end{aligned}
$$


Again, Theorem 2 can be used to show that $\phi(\boldsymbol{x})$ can be replaced by Forall $(\boldsymbol{x})$.

\subsection{Principle of Partial Evaluation}

In a series of papers that gave properties by which major logic programming semantics could be classified, Dix introduced the "(Generalized) Principle of Partial Evaluation" ((G)PPE) [7]. The PPE basically states that a positive occurrence of $P$ can be "unfolded" (i.e., $P$ 's definition can be substituted) if $P$ is defined non-recursively. Here, we recall the weak version of this property.

Definition 6 (Weak PPE [7]). Let $\Delta$ be a ground rule set, and let an atom $P$ occur only positively in $\Delta$. Let $P \leftarrow \varphi_{1}, \ldots, P \leftarrow \varphi_{N}$ be all the rules with head $P$, and assume that none of the $\varphi_{i}$ contains $P$. We denote by $\Delta_{P}$ the rule set obtained from $\Delta$ by deleting all rules with head $P$ and replacing each rule "Head $\leftarrow P \wedge \psi$ " containing $P$ by the rules:

$$
\text { Head } \leftarrow \varphi_{1} \wedge \psi . \quad \ldots \quad \text { Head } \leftarrow \varphi_{N} \wedge \psi .
$$

The weak principle of partial evaluation states that there is a 1-1 correspondence between models of $\Delta_{P}$ and models of $\Delta$ (with removal of $\{P, \neg P\}$ ).

Using Theorem 2, we can show that the stable and well-founded semantics exhibit this property: we rewrite 8 as "Head $\leftarrow\left(\varphi_{1} \vee \cdots \vee \varphi_{N}\right) \wedge \psi$ ". The replacement $\delta=\left\{P \leftarrow \varphi_{1} \vee \cdots \vee \varphi_{N}\right.$. $\}$ then satisfies all conditions of Theorem 2.

In the GPPE, $P$ is allowed to have arbitrary occurrences in $\Delta$, and the substitution of $P$ for its definition need not be applied for each rule containing $P$. On the other hand, the definition of $P$ has to be present both before and after the transformation, making the precise relation with our result unclear. [8] showed that the stable and well-founded semantics satisfy GPPE.

\section{Conclusion and future work}

In this paper, we have only considered the application of Theorem 1 to Logic Programming. However, due to the general, algebraic nature of this result, it can easily be more widely applied. Indeed, all of the main semantics for Autoepistemic Logic and Default Logic can also be given a natural characterization in terms of approximation theory [4]. As such, Theorem 1 can directly be applied to prove similar equivalences for these logics. There, too, such results are useful. For instance, [12] already stresses the importance of being able to replace certain modal subformulas in Autoepistemic Logic by new propositional symbols. Even though Autoepistemic Logic is beyond the scope of this paper, Theorem 1 could be used to prove the correctness of such tranformations.

This paper is part of a larger research effort to develop a general "toolkit" of useful theorems in approximation theory. The aim of this project is to isolate important properties of knowledge representation logics and try to characterize these in the framework of approximation theory. In this paper, we have done 
this for predicate introduction. In [16], we did the same for certain modularity properties, proving an algebraic theorem that generalizes a number of known theorems for Logic Programming, Autoepistemic Logic, and Default Logic.

\section{References}

1. Marcello Balduccini and Michael Gelfond. Diagnostic reasoning with A-Prolog. TPLP, 3(4-5):425-461, 2003.

2. Marc Denecker. Extending classical logic with inductive definitions. In J. Lloyd et al., editor, First International Conference on Computational Logic (CL'2000), volume 1861 of Lecture Notes in Artificial Intelligence, July 2000. Springer.

3. Marc Denecker, Victor Marek, and Mirosław Truszczyński. Approximating operators, stable operators, well-founded fixpoints and applications in nonmonotonic reasoning. In J. Minker, editor, Logic-based Artificial Intelligence, chapter 6, pages 127-144. Kluwer Academic Publishers, 2000.

4. Marc Denecker, Victor Marek, and Mirosław Truszczyński. Uniform semantic treatment of default and autoepistemic logics. In Seventh International Conference on Principles of Knowledge Representation and Reasoning (KR'2000), pages 74-84, Breckenridge, April 11-15 2000. Morgan Kaufman.

5. Marc Denecker, Victor Marek, and Mirosław Truszczyński. Uniform semantic treatment of default and autoepistemic logics. Artificial Intelligence, 143(1):79-122, 2003.

6. Marc Denecker and Eugenia Ternovska. A logic of non-monotone inductive definitions and its modularity properties. In Seventh International Conference on Logic Programming and Nonmonotonic Reasoning (LPNMR'7), 2004.

7. Jürgen Dix. A classification theory of semantics of normal logic programs: Ii. weak properties. Fundam. Inform., 22(3):257-288, 1995.

8. Jürgen Dix and Martin Müller. Partial evaluation and relevance for approximations of stable semantics. In Zbigniew W. Ras and Maria Zemankova, editors, ISMIS, volume 869 of Lecture Notes in Computer Science, pages 511-520. Springer, 1994.

9. Michael Gelfond and Vladimir Lifschitz. The stable model semantics for logic programming. In International Joint Conference and Symposium on Logic Programming (JICSLP'88), pages 1070-1080. MIT Press, 1988.

10. Michael Gelfond and Halina Przymusinska. Towards a theory of elaboration tolerance: Logic programming approach. Journal on Software and Knowledge Engineering, 6(1):89-112, 1996.

11. J.W. Lloyd and R.W. Topor. Making prolog more expressive. Journal of Logic Programming, 1(3):225-240, 1984.

12. V. Wiktor Marek and Miroslaw Truszczyński. Autoepistemic logic. J. ACM, 38(3):588-619, 1991.

13. Tom Schrijvers and Alexander Serebrenik. Improving prolog programs: Refactoring for prolog. In Bart Demoen and Vladimir Lifschitz, editors, ICLP, volume 3132 of Lecture Notes in Computer Science, pages 58-72. Springer, 2004.

14. Allen Van Gelder. The alternating fixpoint of logic programs with negation. Journal of Computer and System Sciences, 47(1):185-221, 1993.

15. Allen Van Gelder, Kenneth A. Ross, and John S. Schlipf. The well-founded semantics for general logic programs. Journal of the ACM, 38(3):620-650, 1991.

16. J. Vennekens, D. Gilis, and M. Denecker. Splitting an operator: Algebraic modularity results for logics with fixpoint semantics. ACM Transactions on computational logic (TOCL), 2006. To appear. 